\title{
UPCROSSING PROBABILITIES FOR STATIONARY GAUSSIAN PROCESSES
}

\author{
BY \\ JAMES PICKANDS III ( $\left.{ }^{(}\right)$
}

1. Introduction. Let $\{X(t),-\infty<t<\infty\}$ be a separable, measurable version of a continuous stationary Gaussian stochastic process. In what follows, it will be assumed, without loss of generality, that

$$
E X(t) \equiv 0, \quad E X^{2}(t) \equiv 1 .
$$

The probability measure associated with the process, then, is completely determined by the covariance function

$$
r(t) \equiv E X(s) X(s+t) .
$$

By stationarity, of course, $r(t)$ does not depend upon $s$.

Upcrossings and their properties have been studied by a number of authors. They have used the following definition. An upcrossing of the level $x$ is said to have occured at $t_{0}$ iff

$$
X\left(t_{0}\right)=x, \text { and } X^{\prime}\left(t_{0}\right)>0,
$$

where $X^{\prime}\left(t_{0}\right)$ is the derivative of the realization $X(t)$ at $t_{0}$. Obviously, such a definition is meaningful only if the realizations are everywhere differentiable with probability one. A necessary condition for this is that

$$
r(t)=1-C t^{2}+o\left(t^{2}\right),
$$

as $t \rightarrow 0$, for some finite positive constant $C$. See for example Cramér [5]. Many processes considered in the literature do not satisfy (1.2). See, for example Slepian [10]. In this paper we introduce a new but natural definition of an upcrossing. For any $\varepsilon>0$, we say that an " $\varepsilon$-upcrossing" of the level $x$ occurs at $t_{0}$ if

$$
X\left(t_{0}\right)=x \text {, and } X(t)<x,
$$

for all $t$ such that $t_{0}-\varepsilon \leqq t<t_{0}$. The advantage of this definition is that it is not necessary to assume that $X(t)$ is differentiable everywhere. It is only necessary to assume that $X(t)$ is continuous everywhere. In order that this be true with probability one, it is sufficient that there exist a $\beta>1$ such that

$$
\limsup _{t \rightarrow 0}|\log t|^{\beta}(1-r(t))<\infty .
$$

Received by the editors January 17, 1969 and, in revised form, February 24, 1969.

(1) Supported by National Science Foundation Grant GP-8716.

Copyright (C) 1969, American Mathematical Society 
See Belyaev [2]. In this paper, it is assumed throughout that

$$
r(t)=1-C t^{\alpha}+o\left(t^{\alpha}\right),
$$

as $t \rightarrow 0$ for some finite positive constant $C$, and $\alpha$ in $(0,2]$. Of course $\alpha$ cannot be greater than 2 , since then $r(t)$ would not be positive definite.

For upcrossings of the conventional type, it has been proved that

$$
\mu \equiv E N(x, t) / t=\sqrt{ } C \exp \left(-x^{2 / 2}\right) / \sqrt{ } 2 \pi,
$$

where $N(x, t)$ is the number of upcrossings in the interval $(0, t]$. As observed above, this expression is only meaningful if $\alpha=2$. See Cramér and Leadbetter [6, pp. 256-271]. In $\S 2$, a limiting form is found for $\mu$, when $\varepsilon$-upcrossings are involved, and (1.3) holds.

It was shown by Volkonski and Rozanov [11] that for any $\lambda, 0<\lambda<\infty$,

$$
\lim _{x \rightarrow \infty} P\{N(x, \lambda / \mu)=k\}=e^{-\lambda} \lambda^{k} / k !
$$

for $k=0,1, \ldots$, provided a uniform mixing condition holds. That condition involves the behavior of $r(t)$ as $t \rightarrow \infty$. It has been pointed out that the upcrossings constitute a regular stationary stream of events. See [6, pp. 222-227]. It is well known that in order that the number of events have the Poisson distribution, it is necessary and sufficient that on any set of nonoverlapping intervals, the numbers of events be mutually independent. This is, of course, not true for the upcrossings of a stationary Gaussian process. It is heuristically clear, though, that if events far removed in time tend to be less dependent, the independence condition may be approached in the limit. Hence the result (1.4). The mixing condition referred to above does indeed involve the weakening of dependence over time. However, it is a rather strict condition and it is quite difficult to verify. Remarking that this is so, Cramér [5] proved that this condition can be replaced by a simpler and weaker one. He assumed that

$$
r(t)=O\left(t^{-\beta}\right)
$$

for some $\beta>0$, as $t \rightarrow \infty$. Heuristically, it would be reasonable to suppose that a similar result would hold for $\varepsilon$-upcrossings. That this is indeed so is the substance of $\S 3$. The result is proved assuming (1.3) and a condition similar in kind to (1.5) but still weaker.

In $\S 4$, it is shown that the limiting distribution of the waiting time is the Gamma distribution. Some other general observations are also contained in $\$ 4$.

By symmetry an $\varepsilon$-downcrossing could be defined as follows. We say that an $\varepsilon$-downcrossing of the level $x$ occurs at $t_{0}$ if an $\varepsilon$-upcrossing of the level $x$ occurs at $-t_{0}$, for the process $X(-t)$. Clearly then, those results which are presented in this paper for $\varepsilon$-upcrossings, hold for $\varepsilon$-downcrossings as well. The most complete compendium on problems involving upcrossings of the conventional kind is, of course, the book by Cramér and Leadbetter [6]. 
2. The expected number of upcrossings. In this section a theorem is stated and proved which gives an asymptotic formula for $\operatorname{EN}(\varepsilon, x, t)$ as $x \rightarrow \infty$ where, of course, $N(\varepsilon, x, t)$ is the number of $\varepsilon$-upcrossings of the level $x$, in the interval from 0 to $t$. First, we define the following quantities. Let

$$
\begin{aligned}
A_{1}(t) & \equiv \inf _{0 \leqq s \leqq t}|s|^{-\alpha}\left(1-r^{2}(s)\right) / 2, \\
A_{2}(t) & \equiv \sup _{0 \leqq s \leqq t}|s|^{-\alpha}\left(1-r^{2}(s)\right) / 2, \\
B(t) & =\inf _{0 \leqq s \leqq t} r(s) .
\end{aligned}
$$

For any $a, x, 0<a, x<\infty$, let the probability measure $P_{x}(\cdot)$ be defined on the process $\{X(t),-\infty<t<\infty\}$ as follows. For any integer $k$, the joint distribution of $\left\{X\left(\right.\right.$ jax $\left.\left.^{-2 / \alpha}\right),-k \leqq j \leqq k\right\}$ is the same under $P_{x}(\cdot)$ as under $P(\cdot)$. For any $t$, let $k$ be the integer such that $k_{a x}-2 / \alpha \leqq t<(k+1) a x^{-2 / \alpha}$. Then, under $P_{x}(\cdot)$ with probability $1, X(t)=X\left(\right.$ kax $\left.^{-2 / \alpha}\right)$.

THEOREM 2.1. Let $\{X(t),-\infty<t<\infty\}$ be a separable measurable version of a stationary Gaussian stochastic process with means 0 , and covariance function $r(t)$, for which (1.3) holds. Assume also that

Then

$$
\inf _{0 \leqq t \leqq \varepsilon}|t|^{-\alpha}(1-r(t))>0 .
$$

where

$$
\lim _{x \rightarrow \infty} E N(\varepsilon, x, t) / x^{2 / \alpha} \Psi(x) t=C^{1 / \alpha} H_{\alpha}
$$

$$
\begin{aligned}
\Psi(x) & \equiv(2 \pi)^{-1 / 2} x^{-1} e^{-x^{2} / 2}, \\
0<H_{\alpha} & \equiv \lim _{T \rightarrow \infty} T^{-1} \int_{0}^{\infty} e^{s} p\left\{\sup _{0 \leqq t \leqq T} Y(t)>s\right\} d s<\infty,
\end{aligned}
$$

and $Y(t)$ is a nonstationary Gaussian process with mean and covariance functions

$$
\begin{aligned}
E Y(t) & =-|t|^{\alpha}, \\
\operatorname{cov}\left(Y\left(t_{1}\right), Y\left(t_{2}\right)\right) & =-\left|t_{1}-t_{2}\right|^{\alpha}+\left|t_{1}\right|^{\alpha}+\left|t_{2}\right|^{\alpha} .
\end{aligned}
$$

Before proceeding to prove the Theorem, several lemmas are stated and proven.

LeMma 2.1. For all $x>0$,

$$
\Psi(x)\left(1-x^{-2}\right) \leqq 1-\Phi(x) \leqq \Psi(x),
$$

where $\Psi(x)$ is given by (2.2), and

$$
\Phi(x)=(2 \pi)^{-1 / 2} \int_{-\infty}^{x} \exp \left(-t^{2} / 2\right) d t .
$$

Furthermore

$$
\lim _{x \rightarrow \infty}(1-\Phi(x)) / \Psi(x)=1 .
$$

Proof. The inequality (2.4) follows immediately by differentiation. The result (2.6) is given in Cramér [4, p. 374]. 
Lemma 2.2. For any $a, 0<a<\infty$, provided that (1.3) holds with $C=1$,

$$
\begin{aligned}
\lim _{x \rightarrow \infty} P_{x}\left\{Z\left(\text { nax }^{-2 / \alpha}\right)>x\right\} / \Psi(x) & =H_{\alpha}(n, a) \\
& \equiv 1+\int_{0}^{\infty} e^{s} p\left\{\max _{0 \leqq k \leqq n} Y(k a)>s\right\} d s<\infty
\end{aligned}
$$

where $\{Y(t), 0 \leqq t<\infty\}$ has the mean and covariance functions (2.3), and

$$
Z(t) \equiv \sup _{0 \leqq s \leqq t} X(s) .
$$

Proof. Obviously, for any $a, b, 0<a, b<\infty$,

$$
\begin{aligned}
P_{x}\left\{Z\left(\operatorname{nax}^{-2 / \alpha}\right)>x\right\}= & P_{x}\{X(o)>x\}+P_{x}\left\{b<X(o) \leqq x, Z\left(\text { nax }^{-2 / \alpha}\right)>x\right\} \\
& +P_{x}\left\{X(o) \leqq b, Z\left(\text { nax }^{-2 / \alpha}\right)>x\right\} .
\end{aligned}
$$

First, let us consider the third term on the right-hand side of (2.9). Clearly, it is dominated by $P_{x}\left\{Z\left(\right.\right.$ nax $\left.\left.^{-2 / \alpha}\right)>x: X(o) \leqq b\right\}$. As is well known, the conditional covariance matrix for the variates $X\left(\operatorname{kax}^{-2 / \alpha}\right), 1 \leqq k \leqq n$, given that $X(o)=u$ is independent of $u$, and the conditional means are $r\left(\mathrm{kax}^{-2 / \alpha}\right) u$. But, by (1.3), for sufficiently large $x$, the covariances are all positive, so that

$$
P_{x}\left\{Z\left(\text { nax }^{-2 / \alpha}\right)>x: X(o)=u\right\}
$$

is a nondecreasing function of $u$. Therefore,

$$
P_{x}\left\{Z\left(\text { nax }^{-2 / \alpha}\right)>x: X(o) \leqq b\right\} \leqq P_{x}\left\{Z\left(\text { nax }^{-2 / \alpha}\right)>x: X(o)=b\right\} .
$$

Furthermore,

$$
\begin{aligned}
P_{x}\left\{Z\left(\text { nax }^{-2 / \alpha}\right)>x: X(o)=b\right\} \\
\qquad \sum_{k=0}^{n} P\left\{X\left(k^{-2 / \alpha}\right)>x: X(o)=b\right\} \\
=\sum_{k=0}^{n}\left(1-\Phi\left(\left(x-r\left(k a x^{-2 / \alpha}\right) b\right) /\left(1-r^{2}\left(k a x^{-2 / \alpha}\right)\right)^{1 / 2}\right)\right) .
\end{aligned}
$$

But, evidently, $\left(1-r^{2}\left(k a x^{-2 / \alpha}\right)\right)^{1 / 2} \sim \sqrt{ } 2(k a)^{\alpha / 2} x^{-1}$, as $x \rightarrow \infty$. Thus, for each $k$,

$$
1-\Phi\left(\left(x-r\left(k a x^{-2 / \alpha}\right) b\right) /\left(1-r^{2}\left(k a x^{-2 / \alpha}\right)\right)^{1 / 2}\right) \leqq 1-\Phi\left(C_{1} x(x-b)\right)=o(1-\Phi(x)),
$$

for some positive finite constant $C_{1}$, as $x \rightarrow \infty$, where $\Phi(x)$ is given by (2.5). Therefore, by Lemma 2.1,

$$
\lim _{x \rightarrow \infty} P_{x}\left\{X(o) \leqq b, Z\left(\text { nax }^{-2 / \alpha}\right)>x\right\} / \Psi(x)=0 .
$$

Now, consider the second term on the right side of (2.9).

$$
\begin{aligned}
P_{x}\{b<X(o) & \left.\leqq x, Z\left(\text { nax }^{-2 / \alpha}\right)>x\right\} \\
& =(2 \pi)^{-1 / 2} \int_{b}^{x} \exp \left(-u^{2} / 2\right) P_{x}\left\{Z\left(\text { nax }^{-2 / \alpha}\right)>x: X(o)=u\right\} d u .
\end{aligned}
$$


Let $u \equiv x+x^{-1} s$. Then

$$
\begin{aligned}
P_{x}\{b< & \left.X(o) \leqq x, Z\left(\text { nax }^{-2 / \alpha}\right)>x\right\} \\
& =\Psi(x) \int_{x(b-x)}^{0} e^{-s-s^{2} / 2 x^{2}} P_{x}\left\{Z\left(\text { nax }^{-2 / \alpha}\right)>x: X(o)=x+x^{-1} s\right\} d s .
\end{aligned}
$$

Let us define the process $Y_{1}(t) \equiv x\left(X\left(t x^{-2 / \alpha}\right)-x\right)$. Given that $X(o)=x+x^{-1} s$, the mean and covariance functions for $Y_{1}(t)$ are

$$
\begin{aligned}
E Y_{1}(t) & \equiv x\left(r\left(t x^{-2 / \alpha}\right)\left(x+x^{-1} s\right)-x\right)=-x^{2}\left(1-r\left(t x^{-2 / \alpha}\right)\right)+r\left(t x^{-2 / \alpha}\right) s \\
& =-|t|^{\alpha}+s+o(1)
\end{aligned}
$$

as $x \rightarrow \infty$, and

$$
\begin{aligned}
\operatorname{cov}\left(Y_{1}\left(t_{1}\right), Y_{1}\left(t_{2}\right)\right) & =x^{2}\left(r\left(\left(t_{2}-t_{1}\right) x^{-2 / \alpha}\right)-r\left(t_{1} x^{-2 / \alpha}\right) r\left(t_{2} x^{-2 / \alpha}\right)\right) \\
& =\left|t_{1}\right|^{\alpha}+\left|t_{2}\right|^{\alpha}-\left|t_{1}-t_{2}\right|^{\alpha}+o(1)
\end{aligned}
$$

as $x \rightarrow \infty$. See Anderson [1, p. 28]. Let $Y(t) \equiv Y_{1}(t)-s$. Then, as $x \rightarrow \infty$, the means and covariances of $Y(t)$ approach limits which are independent of $s$, in particular those given by (2.3). Evidently

$$
X(t)=x^{-1}\left(Y\left(t x^{2 / \alpha}\right)+s\right)+x,
$$

and the event

$$
\left\{Z\left(\text { nax }^{-2 / \alpha}\right)>x\right\}=\left\{\max _{0 \leqq k \leqq n} x^{-1}(Y(k a)+s)+x>x\right\}=\left\{\max _{0 \leqq k \leqq n} Y(k a)>-s\right\},
$$

except on a set of $P_{x}(\cdot)$ measure zero. So

$$
\begin{aligned}
P_{x}\{b<X(0) & \left.\leqq x, Z\left(\text { nax }^{-2 / \alpha}\right)>x\right\} \\
& =\Psi(x) \int_{x(b-x)}^{0} \exp \left(-s-s^{2} / 2 x^{2}\right) P\left\{\max _{0 \leqq k \leqq n} Y(k a)>-s\right\} d s \\
& =\Psi(x) \int_{0}^{x(x-b)} \exp \left(s-s^{2} / 2 x^{2}\right) P\left\{\max _{0 \leqq k \leqq n} Y(k a)>s\right\} d s
\end{aligned}
$$

Clearly, as $s \rightarrow \infty, P\left\{\max _{0 \leqq k \leqq n} Y(k a)>s\right\}=o\left(e^{-2 s}\right)$ as $s \rightarrow \infty$. Thus it follows by the Dominated Convergence Theorem (Loeve [8, p. 125]) that the integral on the right side of (2.12) converges to the integral on the right side of (2.7). Recalling (2.9) and (2.10) the result follows. The lemma is proved.

LEMMA 2.3. Let $X$ and $Y$ be jointly normally distributed with means 0 , variances 1 , and covariance $r$. Then

$$
P\{X>x, Y>x\} \leqq(1+r) \Psi(x)\left(1-\Phi\left(x((1-r) /(1+r))^{1 / 2}\right)\right),
$$

where $\Phi(x)$ is given by (2.5) and $\Psi(x)$ by (2.2).

Proof. By definition,

$P\{X>x, Y>x\}=(2 \pi)^{-1}\left(1-r^{2}\right)^{-1 / 2} \int_{x}^{\infty} \int_{x}^{\infty} \exp \left(-\left(s^{2}+t^{2}-2 r s t\right) / 2\left(1-r^{2}\right)\right) d s d t$ 
Let $\omega \equiv x\left(1-r^{2}\right)^{1 / 2}$, and consider the change of variables $s \equiv x+x^{-1} u, t \equiv x+x^{-1} v$. Then

$$
P\{X>x, Y>x\}=(2 \pi)^{-1} x^{-2} \exp \left(-x^{2} /(1+r)\right)\left(1-r^{2}\right)^{-1 / 2} I(x, r),
$$

where

$$
\begin{aligned}
I(x, r) & =\int_{0}^{\infty} \int_{0}^{\infty} \exp (-(u+v) /(1+r)) \exp \left(-\left(u^{2}+v^{2}-2 r u v\right) / 2 x^{2}\left(1-r^{2}\right)\right) d u d v \\
& \leqq J(x, r) \equiv \int_{0}^{\infty} \int_{0}^{\infty} \exp (-(u+v) /(1+r)) \exp \left(-(u-v)^{2} / 2 \omega^{2}\right) d u d v
\end{aligned}
$$

Since $1 /(1+r)-1 / 2=(1-r) / 2(1+r)$, by $(2.14)$ above,

$$
P\{X>x, Y>x\} \leqq(2 \pi)^{-1 / 2} \omega^{-1} \exp \left(-\omega^{2} / 2(1+r)^{2}\right) \Psi(x) J(x, r) .
$$

Now, let us evaluate $J(x, r)$. Let $s \equiv(u+v) /(1+r), t=(u-v) / \omega$. Then $-\infty<t<\infty$ and $\omega|t| /(1+r) \leqq s<\infty$. The Jacobian $|\partial(s, t) / \partial(u, v)|=2 / \omega(1+r)$. So

$$
d s d t=2 d u d v / \omega(1+r) .
$$

Thus

$$
\begin{aligned}
J(x, r) & \leqq(\omega(1+r) / 2) \int_{-\infty}^{\infty} \exp \left(-t^{2} / 2\right)\left(\int_{\omega|t| /(1+r)}^{\infty} e^{-s} d s\right) d t \\
& =\omega(1+r) \int_{0}^{\infty} \exp \left(-t^{2} / 2\right) \exp (-\omega|t| /(1+r)) d t \\
& =\omega(1+r) \int_{0}^{\infty} \exp \left(-(t+\omega /(1+r))^{2} / 2\right) d t \exp \left(\omega^{2} / 2(1+r)^{2}\right) \\
& =(2 \pi)^{1 / 2} \omega(1+r) \exp \left(\omega^{2} / 2(1+r)^{2}\right)(1-\Phi(\omega /(1+r))),
\end{aligned}
$$

where $\Phi(x)$ is given by (2.5). Combining (2.15) and (2.16), the result (2.13) follows. The lemma is proved.

LeMMA 2.4. If (1.3) holds, with $C=1$, and $A_{1}(t)>0$,

(2.17) $\liminf _{x \rightarrow \infty} P_{x}\{Z(t)>x\} / x^{2 / \alpha} \Psi(x) t \geqq a^{-1}\left(1-4 \sum_{k=0}^{\infty}\left(1-\Phi\left(\left(A_{1}(t)(k a)^{\alpha} / 8\right)^{1 / 2}\right)\right)\right)$, which is greater than 0 for all a, where $A_{1}(t)$ is given in (2.1). The expression on the right side of (2.17) is finite provided $A_{1}(t)>0$.

\section{Proof. Clearly}

$$
\begin{aligned}
P_{x}\{Z(t)>x\} \geqq & \sum_{k=0}^{m} P\left\{X\left(\operatorname{kax}^{-2 / \alpha}\right)>x\right\} \\
& -\sum_{k \neq l=0}^{m} P\left\{X\left(k^{2} x^{-2 / \alpha}\right)>x, X\left(\operatorname{lax}^{-2 / \alpha}\right)>x\right\},
\end{aligned}
$$


where $m \equiv\left[x^{2 / \alpha} t / a\right]$, and $[x]$ is the greatest integer less than or equal to $x$. By stationarity, the equation (2.18) can be rewritten

$$
\begin{aligned}
P_{x}\{Z(t)>x\} \geqq & m P\{X(o)>x\} \\
& -2 \sum_{k=1}^{m-1}(m-k) P\left\{X(o)>x, X\left(k a x^{-2 / \alpha}\right)>x\right\} .
\end{aligned}
$$

But by Lemma 2.3,

$$
P\left\{X(o)>x, X\left(k a x^{-2 / \alpha}\right)>x\right\} \leqq 2 \Psi(x)\left(1-\Phi\left(\left(A_{1}(t)(k a)^{\alpha} / 8\right)^{1 / 2}\right)\right) .
$$

Recalling (2.19), then, the result (2.17) follows. Clearly $a$ can be chosen so that the expression on the right side of (2.17) is positive. The lemma is proved.

LeMma 2.5. If (1.3) holds with $C=1$ and $A_{1}(t)>0$, then

$$
\lim _{x \rightarrow \infty} P_{x}\{Z(t)>x\} / x^{2 / \alpha} \Psi(x) t=H_{\alpha}(a) / a,
$$

where

$$
H_{\alpha}(a) \equiv \lim _{n \rightarrow \infty} H_{\alpha}(n, a) / n>0,
$$

$\Psi(x)$ is given by (2.2), and $H_{\alpha}(n, a)$ by (2.7). The function $H_{\alpha}(a)$ is bounded away from 0 for all sufficiently small $a$.

Proof. For each positive integer $k$, let the event $B_{k} \equiv\left\{X\left(\operatorname{kax}^{-2 / \alpha}\right)>x\right\}$, and for an arbitrary integer $n$, let $A_{k} \equiv \bigcup_{l=(k-1) n+1}^{k n} B_{l}$. Clearly,

$$
P_{x}\{Z(t)>x\} \leqq P\left\{\bigcup_{k=1}^{\left[x^{2} / \alpha_{t / n a]+1}\right.} A_{k}\right\}
$$

where $[x]$ is defined to be the greatest integer less than or equal to $x$. By stationarity $P\left\{A_{k}\right\}=P\left\{A_{1}\right\}$ for all $k, k \geqq 1$. Consequently

$$
P_{x}\{Z(t)>x\} \leqq\left(\left[x^{2 / \alpha} t / n a\right]+1\right) P\left(A_{1}\right)=\left(\left[x^{2 / \alpha} t / n a\right]+1\right) P_{x}\left\{Z\left(\text { nax }^{-2 / \alpha}\right)>x\right\} .
$$

By Lemma 2.2, then,

$$
\limsup _{x \rightarrow \infty} P_{x}\{Z(t)>x\} / x^{2 / \alpha} \Psi(x) t \leqq H_{\alpha}(n, a) / n a,
$$

where $H_{\alpha}(n, a)$ is given by (2.7). On the other hand,

$$
\begin{aligned}
P_{x}\{Z(t)>x\} & \geqq \sum_{k=1}^{\left[x^{2 / \alpha} / n a\right]} P\left(A_{k}\right)-\sum_{k \neq l=1}^{\left[x^{2 / \alpha} / / n a\right]} P\left(A_{k} \cap A_{l}\right) \\
& \geqq\left[x^{2 / \alpha} t / n a\right]\left(P\left(A_{1}\right)-\sum_{k=1}^{\left[x^{2 / \alpha} / / n a\right]} P\left(A_{l} \cap A_{k+1}\right)\right) \\
& =\left[x^{2 / \alpha} t / n a\right]\left(P\left(A_{1}\right)-\sum_{k=1, l=n+1}^{n\left[x^{2 / \alpha} / n a\right]} P\left(B_{k} \cap B_{l}\right)\right) .
\end{aligned}
$$


But $x^{2}\left(1-r^{2}\left(a x^{-2 / \alpha} m\right)\right)>2 A_{1}(t)(m a)^{\alpha}$ where $A_{1}(t)$, given by $(2.1)$, is assumed to be greater than 0 . Recalling Lemma 2.2 ,

(2.23) $\liminf _{x \rightarrow \infty} P_{x}\{Z(t)>x\} / x^{2 / \alpha} \Psi(x) t \geqq\left(H_{\alpha}(n, a)-\sum_{k=1, l=n+1}^{\infty} d_{|k-l|}\right) / n a$,

where

$$
d_{m}=2\left(1-\Phi\left(\left(A_{1}(t)(m a)^{\alpha} / 8\right)^{1 / 2}\right)\right)
$$

Clearly

$$
\sum_{m=1}^{\infty} d_{m}<\infty, \sum_{k=1, l=n+1}^{\infty} d_{|k-l|}=\sum_{k=1}^{n}\left(\sum_{l=k}^{\infty} d_{l}\right) .
$$

But $\lim _{l \rightarrow \infty} \sum_{l=k}^{\infty} d_{l}=0$, by (2.25). But Caesaro convergence implies convergence. So

$$
\lim _{n \rightarrow \infty}\left(\sum_{k=1}^{\infty} d_{l=n+1} d_{|k-l|}\right) / n a=0 .
$$

Combining (2.22), (2.23), and (2.26), we get the result. It follows, by Lemma 2.4, that there exist constants $a_{0}, a_{1}, 0<a_{0}, a_{1}<\infty$, such that for any $a>a_{0}, H_{\alpha}(a)>a_{1}$ $>0$. For any $a$, there exists an integer $m$, such that $a m>a_{0}$. By definition (2.7) of $H_{\alpha}(n, a)$, clearly $H_{\alpha}(n, a m) \geqq H_{\alpha}(n, a)$. Hence $H_{\alpha}(a)>H_{\alpha}(a m)>a_{1}$. So $H_{\alpha}(a)$ is uniformly bounded away from 0 . The lemma is proved.

LEMMA 2.6. Let $Q(x, r, h)$ be the probability that $X \leqq x$, and $Y>x+h$, where $X$ and $Y$ are jointly normally distributed with means 0 , variance 1 , and covariance $r$, and $x, y>0$. Then

$$
Q(x, r, h) \leqq x r^{-1}\left(1-r^{2}\right)^{1 / 2} \Psi(x) R\left(\frac{h r}{\left(1-r^{2}\right)^{1 / 2}}-x\left(\frac{1-r}{1+r}\right)^{1 / 2}\right),
$$

where

$$
R(x)=\int_{x}^{\infty}(1-\Phi(s)) d s,
$$

and $\Phi(x)$ is given by (2.5), and $\Psi(x)$ by (2.2).

Proof. Suppose that $X$ and $Y$ are jointly normally distributed with means 0 , variances 1 , and covariance $r$. Then

$$
\begin{aligned}
P\{X \leqq x: Y=u\} & =P\left\{(X-r u) /\left(1-r^{2}\right)^{1 / 2} \leqq(x-r u) /\left(1-r^{2}\right)^{1 / 2}: Y=u\right\} \\
& =\Phi\left((x-r u) /\left(1-r^{2}\right)^{1 / 2}\right)=\Phi\left(x\left(\frac{1-r}{1+r}\right)^{1 / 2}+r \frac{(x-u)}{\left(1-r^{2}\right)^{1 / 2}}\right)
\end{aligned}
$$

So

$$
Q(x, r, h)=(2 \pi)^{-1 / 2} \int_{x+h}^{\infty} \exp \left(-u^{2} / 2\right) \Phi\left(x\left(\frac{1-r}{1+r}\right)^{1 / 2}+r \frac{(x-u)}{\left(1-r^{2}\right)^{1 / 2}}\right) d u
$$


Let $u=x+x^{-1} t$. Then

$$
\begin{aligned}
Q(x, r, h) & =\Psi(x) \int_{h x}^{\infty} \exp \left(-t-t^{2} / 2 x^{2}\right) \Phi\left(x\left(\frac{1-r}{1+r}\right)^{1 / 2}-r \frac{t}{x\left(1-r^{2}\right)^{1 / 2}}\right) d t \\
& \leqq \Psi(x) \int_{h x}^{\infty} e^{-t}\left(1-\Phi\left(\frac{r t}{x\left(1-r^{2}\right)^{1 / 2}}-x\left(\frac{1-r}{1+r}\right)^{1 / 2}\right)\right) d t .
\end{aligned}
$$

Consider the function

$$
g(y) \equiv \int_{h x}^{\infty} e^{-t}(1-\Phi(y t-c)) d t
$$

Let $s=y t-c$. Then,

$$
\begin{aligned}
g(y) & \equiv y^{-1} \int_{h x y-c}^{\infty} \exp (-(s+c) / y)(1-\Phi(s)) d s \\
& \leqq y^{-1} \int_{h x y-c}^{\infty}(1-\Phi(s)) d s .
\end{aligned}
$$

The result (2.27) follows, letting $y=r / x\left(1-r^{2}\right)^{1 / 2}, c=x((1-r) /(1+r))^{1 / 2}$. The lemma is proved.

Let us now consider the function $R(x)$. By Lemma 2.1, and using integration by parts,

$$
\begin{aligned}
R(x) & =-x(1-\Phi(x))+(2 \pi)^{-1 / 2} \int_{x}^{\infty} s \exp \left(-s^{2} / 2\right) d s \\
& =-x(1-\Phi(x))+(2 \pi)^{-1 / 2} \exp \left(-x^{2} / 2\right) \\
& \leqq(2 \pi)^{-1 / 2} \exp \left(-x^{2} / 2\right)-(2 \pi)^{-1 / 2} x\left(x^{-1}-x^{-3}\right) \exp \left(-x^{2} / 2\right) \\
& =(2 \pi)^{-1 / 2} x^{-2} \exp \left(-x^{2} / 2\right)=x^{-1 \Psi}(x),
\end{aligned}
$$

where $\Psi(x)$ is given by $(2.2)$.

Lemma 2.7. For any $a, b, \gamma, 0<a, \gamma<\infty, 2^{-\alpha / 2}<b<1$, provided (1.3) holds with $C=1$,

$$
\limsup _{x \rightarrow \infty} P\left\{X(o) \leqq x-x^{-1} \gamma, Z\left(a x^{-2 / \alpha}\right)>x\right\} / \Psi(x) \leqq M(a, \gamma),
$$

where

$$
\begin{aligned}
M(a, \gamma) \equiv & (a / 2)^{\alpha / 2} \sum_{k=0}^{\infty} 2^{k(1-(\alpha / 2))} \\
& \cdot R\left(\gamma(1-b)(a / 2)^{-\alpha / 2}\left(2^{\alpha / 2} b\right)^{k}-2^{-1 / 2}(a / 2)^{\alpha / 2} 2^{-\alpha k / 2}\right)
\end{aligned}
$$

and $\Psi(x), R(x)$, and $Z(t)$ are given respectively by (2.2), (2.8), and (2.28).

Proof. The events $D_{k}, k=0,1,2, \ldots$ are defined as follows. Let

$$
\begin{aligned}
& D_{k}=\left\{\max _{0 \leqq j \leqq 2^{k-1}} X\left(j a x^{-2 / \alpha} / 2^{k}\right) \leqq x-x^{-1} \gamma+x^{-1} \gamma(1-b)\right. \\
&\left.\cdot \sum_{j=0}^{k-1} b^{j}=x-x^{-1} \gamma b^{k}, \max _{0 \leqq j \leqq 2^{k+1}} X\left(j a x^{-2 / \alpha} / 2^{k+1}\right)>x-x^{-1} \gamma b^{k+1}\right\} .
\end{aligned}
$$


Clearly,

$$
D_{k} \subset U_{j=0}^{2^{k}-1} E_{j, k}
$$

where

$$
E_{j, k} \equiv\left\{X\left(j a x^{-2 / \alpha} / 2^{k}\right) \leqq x-x^{-1} \gamma b^{k}, X\left((2 j+1) a x^{-2 / \alpha} / 2^{k+1}\right)>x-x^{-1} \gamma b^{k+1}\right\} .
$$

Recalling Lemma 2.6,

$$
P\left\{E_{j, k}\right\}=Q\left(x-x^{-1} \gamma b^{k}, r\left(2^{-(k+1)} a x^{-2 / \alpha}\right), x^{-1} \gamma(1-b) b^{k}\right),
$$

where $Q(x, r, h)$ is given by (2.27). Consider the argument of the term on the right side of (2.27).

$$
\begin{aligned}
\frac{h r}{\left(1-r^{2}\right)^{1 / 2}}-x\left(\frac{1-r}{1+r}\right)^{1 / 2}= & \frac{\gamma(1-b) b^{k} r}{x\left(1-r^{2}\right)^{1 / 2}}-\left(x-x^{-1} \gamma b^{k}\right)\left(\frac{1-r}{1+r}\right)^{1 / 2} \\
\geqq & \frac{\gamma(1-b) b^{k} r}{x\left(1-r^{2}\right)^{1 / 2}}-x\left(\frac{1-r}{1+r}\right)^{1 / 2} \\
\geqq & \gamma(1-b) B\left(2^{-(k+1)} a x^{-2 / \alpha}\right)\left(2 A_{2}\left(2^{-(k+1)} a x^{-2 / \alpha}\right)\right)^{-1 / 2} \\
& \cdot(a / 2)^{-\alpha / 2}\left(2^{\alpha / 2} b\right)^{k}-\left(1+r\left(2^{-(k+1)} a x^{-2 / \alpha}\right)\right)^{-1 / 2} \\
& \cdot\left(A_{2}\left(2^{-(k+1)} a x^{-2 / \alpha}\right)\right)^{1 / 2}(a / 2)^{\alpha / 2} 2^{-\alpha k / 2},
\end{aligned}
$$

where $A_{2}(t)$ and $B(t)$ are given by (2.1). Clearly $\lim _{t \rightarrow 0} B(t)=\lim _{t \rightarrow 0} 2 A_{2}(t)=1$. So, by the Bounded Convergence Theorem, in the limit as $x \rightarrow \infty$, the argument is

$$
\gamma(1-b)(a / 2)^{-\alpha / 2}\left(2^{\alpha / 2} b\right)^{k}-2^{-1 / 2}(a / 2)^{\alpha / 2} 2^{-\alpha k / 2} .
$$

Now consider the term by which the function on the right side of (2.27) is multiplied.

$$
\begin{aligned}
x r^{-1}\left(1-r^{2}\right)^{1 / 2} \leqq & \left(B\left(2^{-(k+1)} a x^{-2 / \alpha}\right)\right)^{-1}\left(2 A_{2}\left(2^{-(k+1)} a x^{-2 / \alpha}\right)\right)^{1 / 2} \\
& \cdot\left(2^{-(k+1)} a\right)^{\alpha / 2} \rightarrow\left(2^{-(k+1)} a\right)^{\alpha / 2} \quad \text { as } x \rightarrow \infty
\end{aligned}
$$

It follows that

$$
\begin{aligned}
\limsup _{x \rightarrow \infty} & P\left\{E_{j, k}\right\} / \Psi(x) \\
& \leqq\left(2^{-(k+1)} a\right)^{\alpha / 2} R\left(\gamma(1-b)(a / 2)^{-\alpha / 2}\left(2^{\alpha / 2} b\right)^{k}-2^{-1 / 2}(a / 2)^{\alpha / 2} 2^{-\alpha k / 2}\right),
\end{aligned}
$$

where $R(y)$ is given by (2.28). But

$$
P\left\{D_{k}\right\} \leqq \sum_{j=0}^{2^{k}-1} P\left\{E_{j, k}\right\}=2^{k} P\left\{E_{1, k}\right\},
$$

since $P\left\{E_{j, k}\right\}$ is the same for all $j$. But

$$
P\left\{X(0) \leqq x-x^{-1} \gamma, Z\left(a x^{-2 / \alpha}\right)>x\right\}<\sum_{k=0}^{\infty} P\left\{D_{k}\right\} .
$$

Combining (2.34), (2.35), and (2.36), we get the result. The lemma is proved. 
Lemma 2.8. For any $a, 0<a<\infty$, if (1.3) holds with $C=1$, then

$$
\limsup _{x \rightarrow \infty} P\left\{X(o) \leqq x, Z\left(a x^{-2 / \alpha}\right)>x\right\} / \Psi(x) \leqq M(a),
$$

where

$$
M(a) \equiv \inf _{0<\gamma<\infty}\left(M(a, \gamma)+e^{\gamma}-1\right)
$$

$\Psi(x)$ is given by (2.2), and $M(a, \gamma)$ by (2.33). Furthermore

$$
\lim _{a \rightarrow 0} M(a) / a=0 .
$$

Proof. Clearly, for any $\gamma, 0<\gamma<\infty$,

$$
\begin{aligned}
P\{X(o) & \left.\leqq x, Z\left(a x^{-2 / \alpha}\right)>x\right\} \\
& \leqq P\left\{X(o) \leqq x-x^{-1} \gamma, Z\left(a x^{-2 / \alpha}\right)>x\right\}+P\left\{x-x^{-1} \gamma<X(o) \leqq x\right\} .
\end{aligned}
$$

By Lemma 2.1

$$
P\left\{X(o)>x-x^{-1} \gamma\right\} \sim e^{\gamma \Psi}(x)
$$

as $x \rightarrow \infty$. Combining (2.39) and (2.40), by Lemma 2.7 , we get the result. It is clear from the definition (2.33) of $M(a, \gamma)$, that (2.38) holds. The lemma is proved.

Lemma 2.9. Provided (1.3) holds with $C=1$, if $A_{1}(t)>0$,

$$
\lim _{x \rightarrow \infty} P\{Z(t)>x\} / x^{2 / \alpha} \Psi(x) t=H_{\alpha}
$$

where

$$
\begin{aligned}
H_{\alpha} & \equiv \lim _{a \rightarrow 0} H_{\alpha}(a) / a=\lim _{T \rightarrow \infty} T^{-1}\left(1+\int_{0}^{\infty} e^{s} P\left\{\sup _{0 \leqq t \leqq T} Y(t)>s\right\} d s\right) \\
& =\lim _{T \rightarrow \infty} T^{-1} \int_{0}^{\infty} e^{s} P\left\{\sup _{0 \leqq t \leqq T} Y(t)>s\right\} d s .
\end{aligned}
$$

Proof. By Lemma 2.8,

$$
\limsup _{x \rightarrow \infty}\left(P\{Z(t)>x\}-P_{x}\{Z(t)>x\}\right) / x^{2 / \alpha} \Psi(x) t \leqq H_{\alpha}-\left(H_{\alpha}(a) / a\right) \leqq M(a) / a,
$$

where $H_{\alpha}, H_{\alpha}(a)$, and $M(a)$ are given respectively by (2.42), (2.21), and (2.37). Let $T$ be an arbitrary finite positive real number, and let $n=[T / a]$. By Lemmas 2.2 and 2.8 ,

$$
\begin{aligned}
\limsup _{x \rightarrow \infty}\left(P\left\{Z\left(\text { nax }^{-2 / \alpha}\right)>x\right\}-P_{x}\right. & \left.\left\{Z\left(\text { nax }^{-2 / \alpha}\right)>x\right\}\right) / n a \Psi(x) \\
& =H_{\alpha}^{\prime}(T)-\left(H_{\alpha}([T / a], a) / a[T / a]\right) \leqq M(a) / a,
\end{aligned}
$$

simultaneously for all $T, 0<T<\infty$, where, of course,

$$
\begin{aligned}
H_{\alpha}^{\prime}(T) & \equiv \limsup _{x \rightarrow \infty} P\left\{Z\left(\text { nax }^{-2 / \alpha}\right)>x\right\} / n a \Psi(x) \\
& =\limsup _{x \rightarrow \infty} P\left\{Z\left(T x^{-2 / \alpha}\right)>x\right\} / \Psi(x) T .
\end{aligned}
$$


Recall Lemma 2.8, and in particular (2.7). Clearly

$$
H_{\alpha}^{\prime}(T)=T^{-1}\left(1+\int_{0}^{\infty} e^{s} P\left\{\sup _{0 \leqq t \leqq T} Y(t)>s\right\} d s\right)
$$

Let $\varepsilon>0$ be arbitrarily chosen. Let $a$ be so chosen that $M(a) / a<\varepsilon / 3$. Let $T^{\prime}$ be so chosen that if $T>T^{\prime}$ then $\left|\left(H_{\alpha}(a) / a\right)-\left(H_{\alpha}([T / a], a) / a[T / a]\right)\right| \leqq \varepsilon / 3$. This is possible by Lemma 2.5 . Now

$$
\begin{aligned}
\left|H_{\alpha}-H_{\alpha}^{\prime}(T)\right| \leqq & \left|H_{\alpha}-\left(H_{\alpha}(a) / a\right)\right|+\left|\left(H_{\alpha}(a) / a\right)-\left(H_{\alpha}([T / a], a) / a[T / a]\right)\right| \\
& +\left|\left(H_{\alpha}([T / a], a) / a[T / a]\right)-H_{\alpha}^{\prime}(T)\right| \\
\leqq & \frac{\varepsilon}{3}+\frac{\varepsilon}{3}+\frac{\varepsilon}{3}=\varepsilon
\end{aligned}
$$

provided $T>T^{\prime}$. So, since $\varepsilon$ was arbitrarily chosen $\lim _{T \rightarrow \infty} H_{\alpha}^{\prime}(T)=H_{\alpha}$. Thus the result (2.41) follows. The lemma is proved.

Proof of Theorem 2.1. Let us begin by assuming that $C=1$. Clearly there exists an $\varepsilon^{\prime}>0$, such that $|t|^{-\alpha}(1-r(t))$ is bounded away from 0 on $\left(0, \varepsilon+\varepsilon^{\prime}\right]$. Let the events $A$ and $B$ be defined as follows. The event $A$ is said to hold if $X(t)>x$ for some $t,-\varepsilon \leqq t \leqq 0$. The event $B$ is said to hold if $X(t)>x$ for some $t, 0 \leqq t \leqq \varepsilon^{\prime}$. By stationarity and by Lemma 2.9,

$$
P\{A \cup B\}=P\left\{Z\left(\varepsilon+\varepsilon^{\prime}\right)>x\right\} \sim H_{\alpha}\left(\varepsilon+\varepsilon^{\prime}\right) x^{2 / \alpha} \Psi(x)
$$

as $x \rightarrow \infty$. Similarly, by Lemma 2.9 ,

and

$$
P\{A\} \sim H_{\alpha} \varepsilon x^{2 / \alpha} \Psi(x),
$$

$$
P\{B\} \sim H_{\alpha} \varepsilon^{\prime} x^{2 / \alpha} \Psi(x)
$$

as $x \rightarrow \infty$. But

$$
P\{A \cap B\}=P\{A\}+P\{B\}-P\{A \cup B\}=o\left(x^{2 / \alpha} \Psi(x)\right)
$$

as $x \rightarrow \infty$. By the definitions,

$$
\left\{N\left(\varepsilon, x, \varepsilon^{\prime}\right)=1\right\} \subset B \subset\left\{N\left(\varepsilon, x, \varepsilon^{\prime}\right)=1\right\} \cup\{A \cap B\},
$$

so

$$
P\left\{N\left(\varepsilon, x, \varepsilon^{\prime}\right)=1\right\} \leqq P(B) \leqq P\left\{N\left(\varepsilon, x, \varepsilon^{\prime}\right)=1\right\}+P(A \cap B) .
$$

Consequently $P(B)-P(A \cap B) \leqq P\left\{N\left(\varepsilon, x, \varepsilon^{\prime}\right)=1\right\} \leqq P(B)$. Recalling (2.44) and (2.45),

$$
P\left\{N\left(\varepsilon, x, \varepsilon^{\prime}\right)=1\right\} \sim H_{\alpha} \varepsilon^{\prime} x^{2 / \alpha} \Psi(x)
$$

as $x \rightarrow \infty$. But, $\varepsilon^{\prime}$ can be chosen to be smaller than $\varepsilon$. Then there can be only one $\varepsilon$-upcrossing on the interval $\left(0, \varepsilon^{\prime}\right]$, and

$$
P\left\{N\left(\varepsilon, x, \varepsilon^{\prime}\right)=1\right\}=E N\left(\varepsilon, x, \varepsilon^{\prime}\right) .
$$


So the theorem is true if $C=1$. Suppose that this is not the case. Let

$$
X_{1}(t) \equiv X\left(t C^{-1 / \alpha}\right)
$$

Then $X_{1}(t)$ satisfies the conditions of the theorem with $C=1$. An $\varepsilon C^{-1 / \alpha}$-upcrossing of the level $x$ by the process $X_{1}(t)$ is an $\varepsilon$-upcrossing of the same level by the process $X(t)$. Furthermore the former satisfies the conditions of the theorem if and only if the latter does. Note that the result of the theorem is independent of the choice of $\varepsilon$. The theorem is proved.

3. The asymptotic distribution. In this section, it is shown that under very general conditions, the Poisson limit theorem holds for " $\varepsilon$-upcrossings" as it does for upcrossings of the conventional kind. The main result of this section is contained in Theorems 3.1 and 3.2. The latter simplifies the conditions for the former.

TheOREM 3.1. Let $\{X(t),-\infty<t<\infty\}$ be a separable, measurable version of a continuous stationary Gaussian process, satisfying the conditions of Theorem 2.1. Assume also that the following two conditions hold.

First, for any $a, \lambda, 0<a, \lambda<\infty$, and any positive integer $n$,

$$
\lim _{x \rightarrow \infty} D_{n}(a, \lambda, x)=0,
$$

where

$$
D_{n}(a, \lambda, x) \equiv \max _{A}\left|P_{x}(A)-P_{x}^{\prime}(A)\right|,
$$

the maximum being taken over all of the sets $A$ which are unions of some of the sets $B_{i}, 1 \leqq i \leqq n$, where $B_{i}$ is the event that an $\varepsilon$-upcrossing of the level $x$ occurs on the interval $((i-1) \lambda / n \mu, i \lambda / n \mu], P_{x}^{\prime}(A)$ is the product measure, and

$$
\mu \equiv E N(\varepsilon, x, t) / t
$$

which has the same value for all positive $t$. Specifically, for each $i$

$$
P_{x}\left(B_{i}\right)=P_{x}^{\prime}\left(B_{i}\right),
$$

but under $P_{x}^{\prime}(\cdot)$, the events $B_{i}$ are mutually independent.

The second condition is that for any such $a, \lambda$, and any $\varepsilon>0$,

$$
\limsup _{x \rightarrow \infty} \sum_{k=1}^{m} Q_{k}(x) \leqq \lambda / a H_{\alpha},
$$

where

$$
\begin{aligned}
l & \equiv\left[\varepsilon x^{2 / \alpha} / a\right], \\
m & \equiv\left[\lambda x^{2 / \alpha} / a \mu\right] \sim\left[\lambda / a H_{\alpha} \Psi(x)\right] \quad \text { as } x \rightarrow \infty, \\
Q_{k}(x) & \equiv\left(1+r_{k}(x)\right)\left(1-\Phi\left(x q_{k}(x)\right)\right), \\
q_{k}(x) & \equiv\left(\left(1-r_{k}(x)\right) /\left(1+r_{k}(x)\right)\right)^{1 / 2},
\end{aligned}
$$

and

$$
r_{k}(x) \equiv r\left(k a x^{-2 / \alpha}\right)
$$


If both of these conditions hold, then, for any $k=0,1,2, \ldots$,

$$
\lim _{x \rightarrow \infty} P\{N(\varepsilon, x, \lambda / \mu)=k\}=e^{-\lambda} \lambda^{k} / k !
$$

Before proceeding to the proof of the theorem, some lemmas are stated and proved.

Lemma 3.1. For any $a, \lambda, 0<a, \lambda<\infty$, if (1.3) holds with $C=1$, and the conditions of Theorem 2.1 hold,

$$
\limsup _{x \rightarrow \infty} P_{x}\{N(\varepsilon, x, \lambda / \mu) \geqq 1\} \leqq \omega(a),
$$

where

$$
\omega(a) \equiv \lambda H_{\alpha}(a) / a H_{\alpha}
$$

and $\mu, H_{\alpha}(a)$, and $H_{\alpha}$ are given respectively by (3.2), (2.21), and (2.42).

Proof. By definition $\{N(\varepsilon, x, \lambda / \mu) \geqq 1\} \subset \bigcup_{k=0}^{[\lambda / \mu]} A_{k}$, where $A_{k}$ is the union of the events $\left\{X\left(\right.\right.$ iax $\left.\left.^{-2 / \alpha}\right)>x\right\},\left[k x^{2 / \alpha} / a\right] \leqq i \leqq\left[(k+1) x^{2 / \alpha} / a\right]$. By Lemma 2.5 and Theorem 2.1, for all $k, \lim _{x \rightarrow \infty} P\left(A_{k}\right) / \mu=H_{\alpha}(a) / a H_{\alpha}=\omega(a) / \lambda$. But $P\{N(\varepsilon, x, \lambda / \mu) \geqq 1\} \leqq$ $([\lambda / \mu]+1) P\left(A_{1}\right) \sim \omega(a)$, as $x \rightarrow \infty$, where $\omega(a)$ is given by (3.10). The lemma is proved.

LEMMA 3.2. If for any $a, \lambda, 0<a, \lambda<\infty$, the condition (3.3) holds, if (1.3) holds with $C=1$, and the conditions of Theorem 2.1 hold,

$$
\limsup _{x \rightarrow \infty} P_{x}\{N(\varepsilon, x, \lambda / \mu) \geqq 2\} \leqq \lambda^{2} / a^{2} H_{\alpha}^{2}
$$

where $\mu$ is given by (3.2), and $H_{\alpha}$ is given by (2.42).

Proof. By definition

$$
P_{x}\{N(\varepsilon, x, \lambda / \mu) \geqq 2\} \leqq \sum_{i, j=0,|i-j| \geqq l}^{m} P_{|i-j|}(x),
$$

where $l$ and $m$ are given by (3.4) and (3.5), respectively, and $P_{k}(x)=P\{X>x, Y>x\}$, $X$ and $Y$ being normally distributed with means 0 , variances 1 , and covariance $r_{k}(x)$ given by (3.8). By Lemma 2.3,

$$
P_{k}(x) \leqq \Psi(x) Q_{k}(x),
$$

where $\Psi(x)$ is given by (2.2), and $Q_{k}(x)$ by (3.6). Recalling (3.12),

$$
P_{x}\{N(\varepsilon, x, \lambda / \mu) \geqq 2\} \leqq 2 m \Psi(x) \sum_{k=1}^{m} Q_{k}(x)
$$

Recalling (3.3), (3.5), then the result (3.11) follows. The lemma is proved. 
Lemma 3.3. If for all $a, \lambda, 0<a, \lambda<\infty$, the condition (3.3) holds, then, if (1.3) holds with $C=1$, and the conditions of Theorem 2.7 hold,

$$
\liminf _{x \rightarrow \infty} P_{x}\{N(\varepsilon, x, \lambda / \mu) \geqq 1\} \geqq \omega(a)-\lambda^{2} / a^{2} H_{\alpha}^{2}
$$

as $\lambda \rightarrow \infty$, where $\omega($ a) is given by (3.10).

Proof. Clearly

$$
\begin{aligned}
P_{x}\{N(\varepsilon, x, \lambda / \mu) \geqq 1\} & \geqq \sum_{k=0}^{[\lambda / \mu]} P\left(A_{k}\right)-\sum_{i, j=0,|i-j| \geqq l}^{[\lambda / \mu]} P\left(A_{i} \cap A_{j}\right) \\
& \geqq[\lambda / \mu] P\left(A_{l}\right)-\sum_{i, j=0,|i-j| \geqq l}^{m} P_{|i-j|}(x) \\
& =[\lambda / \mu] P\left(A_{l}\right)-m \Psi(x) \sum_{k=1}^{m} Q_{k}(x),
\end{aligned}
$$

where $l, m$, and $Q_{k}(x)$ are given respectively by (3.4), (3.5), and (3.6) and the events $A_{k}$ are as defined in Lemma 3.1, by the reasoning of Lemma 3.2. Combining the results of the previous two lemmas, we get the result (3.14). The lemma is proved.

Lemma 3.4. Let the conditions of Theorem 3.1 be satisfied. Then for any $a, \lambda$, $0<a, \lambda<\infty$, if $C=1$,

$$
\lim _{x \rightarrow \infty} P_{x}\{N(\varepsilon, x, \lambda / \mu)=k\}=e^{-\omega(a)} \omega^{k}(a) / k !, \quad 0 \leqq k<\infty,
$$

where $\omega(a), H_{\alpha}(a)$, and $H_{\alpha}$ are given respectively by (3.10), (2.21), and (2.42).

Proof. Let $n$ be an arbitrary positive integer. Let $a$ and $\lambda$ be so chosen that $0<a, \lambda<\infty$. Let the events $B_{i}, 1 \leqq i \leqq n$ be defined as in the statement of the theorem. Let $N(1, n)$ be the number of the events $B_{i}$ which occur. That is, $N(1, n)$ is the number of subintervals on each of which at least one $\varepsilon$-upcrossing occurs. The $n$ subintervals are, of course, the intervals $((i-1) \lambda / n \mu, i \lambda / n \mu], 1 \leqq i \leqq n$. Under the measure $P_{x}^{\prime}(\cdot)$, the generating function for $N(1, n)$ is

$$
Q_{x}^{\prime}(s) \equiv E_{x}^{\prime} s^{N(1, n)}=\prod_{i=1}^{n}\left(1+P_{x}^{\prime}\left(B_{i}\right)(s-1)\right)^{n},
$$

where of course $E_{x}^{\prime}$ denotes expectation with respect to the measure $P_{x}^{\prime}(\cdot)$. Since the events $B_{i}$ are finite in number, if $n$ is held fixed, by the condition (3.1), for all $s$,

$$
\lim _{x \rightarrow \infty}\left|Q_{x}(s)-Q_{x}^{\prime}(s)\right|=0,
$$

where $Q_{x}(s)$ is the same generating function, but under $P_{x}(\cdot)$. But, by Lemmas 3.1 and 3.3 , for any $i, 1 \leqq i \leqq n$

$$
\liminf _{x \rightarrow \infty} P\left(B_{i}\right) \geqq(\omega(a) / n)-O(\omega(a) / n)^{2}
$$


as $n \rightarrow \infty$, and

$$
\limsup _{x \rightarrow \infty} P\left(B_{i}\right) \leqq(\omega(a) / n),
$$

where $\omega(a)$ is given by (3.10). By Lemma 3.2,

$$
E N(2, n)=n O(\omega(a) / n)^{2},
$$

where $N(2, n)$ is the number of intervals on each of which at least two $\varepsilon$-upcrossings occur. Recalling all of these conclusions, the result (3.15) follows. The lemma is proved.

Proof of Theorem 3.1. First, assume $C=1$. Let the event $A$ be the event that the two measures $P(\cdot)$ and $P_{x}(\cdot)$ assign different values to $N(\varepsilon, x, \lambda / \mu)$. Clearly $A \subset \bigcup_{k=0}^{m} B_{k}$, where $B_{k}$ is the event that $X\left(k^{2 a x}{ }^{-2 / \alpha}\right) \leqq x$, and $X(t)>x$ for some $t$ in the interval $\left(k a x^{-2 / \alpha},(k+1) a x^{-2 / \alpha}\right)$. But $P(A) \leqq \sum_{k=0}^{m} P\left(B_{k}\right)=(m+1) P\left(B_{1}\right)$, by stationarity, and, by Lemma 2.8 ,

$$
\limsup _{x \rightarrow \infty} P(A) \leqq \lambda M(a) / a H_{\alpha} \rightarrow 0
$$

as $a \rightarrow 0$, by (2.38), where $M(a)$ is given by (2.37). Recalling (2.42), it follows that $\lim _{a \rightarrow 0} \omega(a)=\lambda$, where $\omega(a)$ is given by (3.10). The theorem is proved, provided $C=1$. It follows in general by the transformation (2.46). The theorem is proved.

THEOREM 3.2. The conclusion of Theorem 3.1 is valid if (1.1) and (1.3) hold, and either

$$
\lim _{t \rightarrow \infty} r(t) \log t=0
$$

or

$$
\int_{-\infty}^{\infty} r^{2}(t) d t<\infty
$$

The proof of this theorem is essentially contained in the following sequence of lemmas.

LEMMA 3.5. Let $c$ be any real positive finite quantity, and let $P(\cdot)$ and $P^{\prime}(\cdot)$ be two normalized multivariate Gaussian measures. That is $P(\cdot)$ and $P^{\prime}(\cdot)$ are measures under which the components are jointly normally distributed with means 0 , and variances 1 . Let the covariances be, respectively, $r_{i j}$ and $r_{i j}^{\prime}$. Then

$$
\left|P\left\{\bigcup_{k=1}^{n}\left(X_{k}>c\right)\right\}-P^{\prime}\left\{\bigcup_{k=1}^{n}\left(X_{k}>c\right)\right\}\right| \leqq D_{n}=\sum_{i, j=1}^{n=1}\left|r_{i j}-r_{i j}^{\prime}\right| \phi\left(c,\left|r_{i j}^{\prime \prime}\right|\right),
$$

where

$$
\phi\left(c,\left|r_{i j}^{\prime \prime}\right|\right) \equiv\left(1-r_{i j}^{\prime \prime 2}\right)^{-1 / 2} \exp \left\{-c^{2} /\left(1+\left|r_{i j}^{\prime \prime}\right|\right)\right\}
$$

and $r_{i j}^{\prime \prime} \equiv \max \left(r_{i j}, r_{i j}^{\prime}\right)$. 
This lemma was originally proved by Berman [3] for the case in which both measures are stationary. The present lemma was first given in [9].

LEMMA 3.6. Simultaneously for all $m \geqq 1$ if (1.3) holds,

$$
\frac{1}{m} \sum_{k=1}^{m}\left|r_{k}(x)\right|=\frac{1}{T} \int_{0}^{T}|r(t)| d t+o\left(a x^{-2 / \alpha}\right),
$$

as $x \rightarrow \infty$, where $r_{k}(x)$ is given by (3.7) and $T=\max ^{-2 / \alpha}$.

Proof. By definition (3.7),

$$
\begin{aligned}
\sum_{k=1}^{m}\left|r_{k}(x)\right|= & \sum_{k=1}^{m}\left|r\left(k a x^{-2 / \alpha}\right)\right| \\
= & \left(x^{2 / \alpha} / a\right) \sum_{k=1}^{m} \int_{(k-1) a x^{-2 / \alpha}}^{k a x^{-2 / \alpha}} d s\left|r\left(k a x^{-2 / \alpha}\right)\right| \\
\leqq & \geqq)\left(x^{2 / \alpha} / a\right) \sum_{k=1}^{m} \int_{(k-1) a x^{-2 / \alpha}}^{k a x^{-2 / \alpha}}|r(s)| d s \\
& +(-)\left(x^{2 / \alpha} / a\right) \sum_{k=1}^{m} \int_{(k-1) a x^{-2 / \alpha}}^{k a x^{-2 / \alpha}}\left|r(s)-r\left(k a x^{-2 / \alpha}\right)\right| d s
\end{aligned}
$$

But by the increments inequality (Loeve [8, p. 195]), and by stationarity

$$
\int_{(k-1) a x^{-2 / \alpha}}^{k a x^{-2 / \alpha}}\left|r(s)-r\left(k a x^{-2 / \alpha}\right)\right| d s \leqq 2 \int_{0}^{a x-2 / \alpha}(1-r(s)) d s .
$$

Therefore

$\left|\frac{1}{m} \sum_{k=1}^{m}\right| r_{k}(x)\left|-\left(x^{2 / \alpha} / a m\right) \int_{0}^{\max -2 / \alpha}\right| r(s)|d s|=2 \int_{0}^{a x-2 / \alpha}(1-r(s)) d s=o\left(a x^{-2 / \alpha}\right)$

as $x \rightarrow \infty$, uniformly in $m$. The lemma is proved.

LeMMA 3.7. If $\int_{-\infty}^{\infty} r^{2}(t) d t<\infty$, then $\lim _{t \rightarrow \infty} r(t)=0$.

This is Lemma 4.8 of [9].

LEMMA 3.8. In order that the condition (3.1) hold, it is sufficient that either (3.21) or (3.22) hold.

Proof. Let $f(x)$ be a nonnegative function such that

$$
\lim _{x \rightarrow \infty} f(x)=\infty, \quad \lim _{x \rightarrow \infty} x^{2 / \alpha} f(x) \Psi(x)=0 .
$$

Let $\varepsilon>0$ be arbitrarily chosen. For sufficiently large $x$,

$$
f(x) /\left(\lambda / H_{\alpha} x^{2 / \alpha} \Psi(x)\right)=H_{\alpha} x^{2 / \alpha} f(x) \Psi(x) / \lambda \leqq \varepsilon .
$$

By Lemma 2.9, then $\lim \sup _{x \rightarrow \infty} P\{Z(f(x))>x\} \leqq \varepsilon$. Since $\varepsilon$ was arbitrarily chosen,

$$
\lim _{x \rightarrow \infty} P\{Z(f(x))>x\}=0 .
$$


By stationarity, it follows that, in the limit as $x \rightarrow \infty$, the probability approaches 0 that $X(t)>x$ on any of the intervals

$$
\left((j-1) \lambda / k H_{\alpha} x^{2 / \alpha} \Psi(x),\left((j-1) \lambda / k H_{\alpha} x^{2 / \alpha} \Psi(x)\right)+f(x)\right), \quad j=1,2, \ldots, k .
$$

Hence the same is true under $P_{x}(\cdot)$. We can, then, "chop out" the above intervals, without loss of generality. So, by Lemma 3.5,

$$
D_{n}(a, \lambda, x) \leqq m \sum_{k=1}^{m}\left|r_{k}(x)\right| \phi\left(x,\left|r_{k}(x)\right|\right)
$$

where $l$ is the largest integer, such that $l a x^{-2 / \alpha} \leqq f(x)$, that is $l \equiv\left[x^{2 / \alpha} f(x) / a\right], m$ is the largest integer, such that

$$
\max ^{-2 / \alpha} \leqq \lambda / H_{\alpha} x^{2 / \alpha} \Psi(x),
$$

that is

$$
m \equiv\left[\lambda / a H_{\alpha} \Psi(x)\right]
$$

and $\phi(x, r)$ is given by (3.23). The condition (3.21) obviously implies that

$$
\lim _{t \rightarrow \infty} r(t)=0 \text {. }
$$

By Lemma 3.7, it also follows from (3.22). Recalling (3.25) it follows that

$$
\sup _{1 \leqq k \leqq \infty}\left|r_{k}(x)\right| \rightarrow 0
$$

as $x \rightarrow \infty$. So, without loss of generality, we can replace the term $\left(1-r_{k}^{2}(x)\right)^{-1 / 2}$ with any constant $C_{1}$ which is greater than 1 . That is, for sufficiently large $x$,

$$
\begin{aligned}
D_{n}(a, \lambda, x) & \leqq C_{1} m \sum_{k=1}^{m}\left|r_{k}(x)\right| \exp -x^{2} /\left(1+\left|r_{k}(x)\right|\right) \\
& =C_{1} m \sum_{k=1}^{m}\left|r_{k}(x)\right|\left(\exp -x^{2} / 2\right)^{2 /\left(1+\left|r_{k}(x)\right|\right)} \\
& =C_{2} m \sum_{k=1}^{m}\left|r_{k}(x)\right| x^{2 /\left(1+\left|r_{k}(x)\right|\right)}(\Psi(x))^{2 /\left(1+\left|r_{k}(x)\right|\right)},
\end{aligned}
$$

where $C_{2}$ is another finite positive constant. Recalling the definition (3.27) of $m$, there exists a finite constant $C_{3}$, such that for all sufficiently large $x$,

$$
D_{n}(a, \lambda, x) \leqq C_{3} m^{-1} \sum_{k=1}^{m}\left|r_{k}(x)\right| x^{2 /\left(1+\left|r_{k}(x)\right|\right)} m^{2\left|r_{k}(x)\right| /\left(1+\left|r_{k}(x)\right|\right)}
$$

First, suppose that (3.21) holds. Let

$$
\delta(t) \equiv \sup _{t \leqq s<\infty}|r(s)|
$$

Then

$$
\delta(t) \log t \leqq \sup _{t \leqq s<\infty} r(s) \log s \rightarrow 0
$$


as $t \rightarrow \infty$. But

$$
\begin{aligned}
m^{2\left|r_{k}(x)\right| /\left(1+\left|r_{k}(x)\right|\right)} & =\exp \left(2 \log m\left|r_{k}(x)\right| /\left(1+\left|r_{k}(x)\right|\right)\right) \\
& \leqq \exp \left(2 \delta\left((l+1) a x^{-2 / \alpha}\right) \log m\right)
\end{aligned}
$$

for all $k \geqq l+1$. But

$$
\delta\left((l+1) a x^{-2 / \alpha}\right) \log m \leqq C_{4} \delta(f(x)) \log (1 / \Psi(x)),
$$

for some finite positive constant $C_{4}$. The function $f(x)$ is arbitrary in the sense that any function can be chosen which satisfies the restrictions (3.25). Let

$$
f(x)=(\Psi(x))^{-1 / 2} .
$$

It is clear that the conditions (3.25) are satisfied. But

$$
\begin{aligned}
\delta(f(x)) \log (1 / \Psi(x)) & =\delta(f(x)) \log \left((f(x))^{2}\right) \\
& =2 \delta(f(x)) \log f(x) \rightarrow 0
\end{aligned}
$$

as $x \rightarrow \infty$, by (3.32). Clearly,

$$
x^{2 /\left(1+r_{k}(x)\right)} \leqq x^{2}=o(\log m)
$$

as $x \rightarrow \infty$. Recalling (3.31) through (3.36),

$$
\begin{aligned}
& m^{-1} \sum_{k=1}^{m}\left|r_{k}(x)\right| x^{2 /\left(1+\left|r_{k}(x)\right|\right)} m^{2\left|r_{k}(x)\right| /\left(1+\left|r_{k}(x)\right|\right)} \\
& \leqq \delta(f(x)) \log \left((f(x))^{2}\right) o(\log m) \exp \delta(f(x)) \log \left((f(x))^{2}\right) \rightarrow 0
\end{aligned}
$$

as $x \rightarrow \infty$. So, it has been shown that the condition (3.21) is sufficient.

Now consider the condition (3.22). Let $\beta, 0<\beta<1 / 2$ be arbitrarily chosen. Provided $x$ (hence $f(x)$ ) is sufficiently large, by (3.30) $\sup _{1 \leqq k<\infty}\left|r_{k}(x)\right| \leqq \beta$, and $\sup _{1 \leqq k<\infty} x^{2 /\left(1+\left|r_{k}(x)\right|\right)} m^{2\left|r_{k}(x)\right| /\left(1+\left|r_{k}(x)\right|\right)} \leqq m^{\beta}$. So, by Lemma 3.6,

$$
\begin{aligned}
D_{n}(a, \lambda, x) & \leqq C_{1} m^{\beta-1} \sum_{k=1}^{m}\left|r_{k}(x)\right| \leqq\left(x^{2 / \alpha} / a\right)^{\beta} T^{\beta-1} \int_{0}^{T}|r(t)| d t+o\left(a x^{-2 / \alpha}\right)^{1-\beta} \\
& \leqq T^{2 \beta-1} \int_{0}^{T}|r(t)| d t+o\left(a x^{-2 / \alpha}\right)^{1-\beta} .
\end{aligned}
$$

So it is sufficient to prove that there exists a $\beta>0$, such that

$$
\lim _{T \rightarrow \infty} T^{\beta-1} \int_{0}^{T}|r(t)| d t=0
$$

But, by the Cauchy-Schwarz inequality,

$$
\left(T^{\beta-1} \int_{0}^{T}|r(t)| d t\right)^{2} \leqq T^{2 \beta-1} \int_{0}^{T} r^{2}(t) d t \rightarrow 0
$$

as $T \rightarrow \infty$, for any $\beta, 0<\beta<1 / 2$. The lemma is proved. 
LEMMA 3.9. If

$$
\lim _{T \rightarrow \infty} \frac{\log T}{T} \int_{0}^{T} r(t)=0
$$

and

$$
\lim _{t \rightarrow \infty} r(t)=0,
$$

then the condition (3.3) of Theorem 3.1 holds.

Proof. Let $\varepsilon^{\prime}>0$ be arbitrarily chosen. Let $t_{0}$ be so chosen that if $t \geqq t_{0}, r(t)<\varepsilon^{\prime}$. This is possible, since it is assumed that (3.37) holds. Let $m^{\prime}$ be the smallest integer such that $\left(m^{\prime}+1\right) a x^{2 / \alpha} \geqq t_{0}$. That is

$$
m^{\prime} \equiv\left[x^{2 / \alpha} t_{0} / a\right]
$$

Then, we decompose the sum (3.3) as follows.

$$
\sum_{k=l}^{m} Q_{k}(x)=\sum_{k=l}^{m^{\prime}} Q_{k}(x)+\sum_{k=m^{\prime}+1}^{m} Q_{k}(x)
$$

where $l, m$, and $m^{\prime}$ are given respectively by (3.4), (3.5), and (3.39). By the assumption (3.37)

$$
\delta=\sup _{\varepsilon \leqq t<\infty} r(t)<1
$$

Then

$$
\sum_{k=l}^{m^{\prime}} Q_{k}(x) \leqq\left(\left[x^{2 / \alpha}\left(t_{0}-\varepsilon\right) / a\right]+1\right)(1-\Phi(x \delta)) \rightarrow 0
$$

as $x \rightarrow \infty$. Now consider the second term on the right side of (3.40). Let $\theta$ be any real number, $0<\theta<1$. By Lemma 2.1, if $x>0$,

$$
\begin{aligned}
(1-\Phi(\theta x)) & \leqq \Psi(\theta x)=\theta^{-1} x^{\theta-1}(\Psi(x))^{\theta} \leqq \theta^{-1} x^{\theta-1}(1-\Phi(x))^{\theta}\left(1-x^{-2}\right)^{-\theta} \\
& \leqq \theta^{-1}(1-\Phi(x))^{\theta}\left(1-x^{-2}\right)^{-\theta}
\end{aligned}
$$

Consequently,

$$
\begin{aligned}
\sum_{k=m^{\prime}+1}^{m} Q_{k}(x) \leqq\left(1+\varepsilon^{\prime}\right) \sum_{k=m^{\prime}+1}^{m}(1-\Phi(x))^{q_{k}(x)} & \\
= & \left(1+\varepsilon^{\prime}\right)\left(\left(\lambda / a H_{\alpha}\right)+o(1)\right)+(1-\Phi(x)) \sum_{k=m^{\prime}+1}^{m}\left((1-\Phi(x))^{q_{k}(x)-1}-1\right)
\end{aligned}
$$

as $x \rightarrow \infty$. But since $\varepsilon^{\prime}$ was arbitrarily chosen, it will suffice to prove that

$$
\lim _{x \rightarrow \infty}(1-\Phi(x)) \sum_{k=m^{\prime}+1}^{m}\left((1-\Phi(x))^{q_{k}(x)-1}-1\right)=0 .
$$

But, clearly, there exists a finite positive constant $C_{1}$ which is such that, recalling 
the definition (3.7) of $q_{k}(x),(1-\Phi(x))^{q_{k}(x)-1}-1 \leqq C_{1}(-\log (1-\Phi(x))) r_{k}(x)$, provided $r_{k}(x) \leqq \delta$, where $\delta$ is given by (3.41). But then it is sufficient to prove that

$$
\lim _{x \rightarrow \infty} \frac{\log m}{m} \sum_{k=1}^{m} r_{k}(x)=0,
$$

where $m$ is given by (3.4). But by Lemma 3.6, the condition (3.43) is equivalent to (3.37). The lemma is proved.

LEMMA 3.10. If either of the conditions (3.21) or (3.22) holds, then the conditions (3.37) and (3.38) hold.

Proof. First, assume that (3.21) holds. Clearly, then, (3.38) does. Now, it is shown that (3.37) follows. Consider the fact that

$$
\frac{\log T}{T} \int_{0}^{T} r(t)=\frac{\log T}{T} \int_{0}^{\sqrt{ } T} r(t) d t+\frac{\log T}{T} \int_{\sqrt{ } T}^{T} r(t) d t .
$$

Consider the first term on the right side of (3.44).

$$
\frac{\log T}{T} \int_{0}^{\sqrt{ } T} r(t) d t \leqq \frac{\log T}{\sqrt{ } T} \rightarrow 0
$$

as $T \rightarrow \infty$. Now consider the second term. Let $\varepsilon>0$ be arbitrarily chosen. Clearly if $T$ is sufficiently large $r(t)<\varepsilon / \log t$, whenever $t>\sqrt{ } T$. So

$$
\begin{aligned}
\frac{\log T}{T} \int_{\sqrt{ } T}^{T} r(t) d t & \leqq \frac{\varepsilon \log T}{T} \int_{\sqrt{ } T}^{T} d t / \log t \\
& \leqq \varepsilon(T-\sqrt{ } T) \log T / T \log \sqrt{ } T \leqq 2 \varepsilon
\end{aligned}
$$

But $\varepsilon>0$ was arbitrarily chosen. So (3.37) holds.

Now, assume that (3.22) holds. By Lemma 3.7, (3.38) holds. By the CauchySchwarz inequality

$$
\frac{\log T}{T} \int_{0}^{T} r(t) \leqq \frac{\log T}{\sqrt{ } T}\left(\int_{0}^{T} r^{2}(t) d t\right)^{1 / 2} \rightarrow 0
$$

as $T \rightarrow \infty$. The lemma is proved.

4. Discussion and special cases. Let $T_{k}$ be the waiting time until the $k$ th $\varepsilon$ upcrossing. The event $\left\{T_{k} \leqq t / \mu\right\}$ is the event that at least $k \varepsilon$-upcrossings will have occurred during the interval $(0, t / \mu)$. Therefore

$$
\lim _{x \rightarrow \infty} P\left\{T_{k} \leqq t / \mu\right\}=F_{k}(t),
$$

where $\mu$ is given by (3.2) and $F_{k}(t) \equiv 1-e^{-t} \sum_{j=0}^{k-1} t^{j} / j !$, provided that the conditions of Theorems 3.1 and 3.2 hold. By differentiation

$$
F_{k}(t)=\frac{1}{(k-1) !} \int_{0}^{t} s^{k-1} e^{-s} d s
$$


which is the Gamma distribution function. This reasoning is essentially the same as that giving the analogous result for upcrossings of the conventional type. See Cramér and Leadbetter [6, pp. 272-273].

It is natural to wonder whether the conditions (3.21), and (3.22) can be substantially weakened. Might the results of Theorems 3.1 and 3.2 hold if (1.3) is true and (3.38) holds. A similar question was raised and answered negatively for stationary Gaussian sequences in $\S 2$ of [9]. For that reason, it is strongly conjectured that (3.38) is not sufficient.

Comparing Theorem 2.1 with Lemma 4.4 of [9], it is obvious that $H_{1}=1$. The referee has pointed out that this can be verified as follows. When $\alpha=1$ we can write $Y(t)=-t+\sqrt{ } 2 W(t)$, where $W(t)$ is a standard Wiener process. That $H_{1}=1$ is evident from well-known properties or. $W(t)$.

Suppose $\alpha=2$. Assume, also, that additional conditions are satisfied, which are sufficient in order that the number of conventional upcrossings has the Poisson distribution in the limit as $x \rightarrow \infty$. Since both types of upcrossings are "widely spaced" in the limit it is obvious that in the limit as $x \rightarrow \infty$, the probability that a given $\varepsilon$-upcrossing is also a conventional one approaches 1 , as does the probability that a conventional upcrossing is an $\varepsilon$-upcrossing. But the exact expected number of conventional upcrossings is

$$
E N(x, t)=\left(2^{-1 / 2} t / \pi\right) \exp \left(-x^{2} / 2\right)
$$

See Cramér and Leadbetter [6, pp. 194 and 197]. So $H_{2}=1 / \sqrt{ } \pi$. It was pointed out by the referee that this is an obvious consequence of the fact that when $\alpha=2$, $Y(t)=-t+2^{1 / 2} U t$ where $U$ is a standard Gaussian variate.

The above equivalence confirms the unsurprising fact that the results do not depend on $\varepsilon$. Suppose that $0<\varepsilon_{1}<\varepsilon_{2}<\infty$. Then an $\varepsilon_{2}$-upcrossing is certainly an $\varepsilon_{1}$-upcrossing, but in the limit as $x \rightarrow \infty$, the probability approaches 1 that an $\varepsilon_{1}$-upcrossing is also an $\varepsilon_{2}$-upcrossing, if Theorem 3.1 holds.

The conditions considered in this paper are of two types. The condition (1.3) is a "local condition" in that it involves the behavior of $r(t)$ as $t \rightarrow 0$. The other conditions, in particular (3.21), (3.22), and (3.38) are "mixing conditions" in the sense that they involve the behavior of $r(t)$ as $t \rightarrow \infty$. All of the conditions have been expressed in terms of the covariance function $r(t)$. It is of interest to consider conditions in terms of the spectral distribution function $F(\omega)$ defined by the relation

$$
r(t)=\int_{-\infty}^{\infty} \cos \omega t d F(\omega) .
$$

In order that the local condition (1.3) hold for $0<\alpha<2$ it is necessary and sufficient that there exist a positive finite constant $C_{1}$, such that

$$
\lim _{\omega \rightarrow \infty} \omega^{\alpha}(1-F(\omega))=\lim _{\omega \rightarrow \infty} \omega^{\alpha} F(-\omega)=C_{1} .
$$


See Kolmogorov and Gnedenko [7, pp. 180-182]. If $\alpha=2$ it is necessary and sufficient that

$$
\int_{-\infty}^{\infty} \omega^{2} d F(\omega)<\infty
$$

The relationships between the mixing conditions, and conditions on $F(\omega)$ are discussed at the end of $\S 4$ of [9]. That discussion suffices for this work as well. From the discussion of the "uniform mixing condition", we can conclude that it is sufficient for the result of Theorems 3.1 and 3.2.

\section{REFERENCES}

1. T. W. Anderson, An introduction to multivariate statistical analysis, Wiley, New York, 1958.

2. Yu. K. Belyaev, Continuity and Hölder's conditions for sample functions of stationary Gaussian processes, Proc. Fourth Berkeley Symposium on Mathematical Statistics and Probability, Vol. II, 1961, pp. 23-33.

3. S. M. Berman, Limit theorems for the maximum term in stationary sequences, Ann. Math. Statist. 35 (1964), 502-516.

4. H. Cramér, Mathematical methods of statistics, Princeton Univ. Press, Princeton, N. J., 1951.

5. - On the intersections between the trajectories of a normal stationary stochastic process and a high level, Ark. Mat. 6 (1966), 337-349.

6. H. Cramér and M. R. Leadbetter, Stationary and related stochastic processes; sample function properties and their applications, Wiley, New York, 1955.

7. A. N. Kolmogorov and B. V. Gnedenko, Limit distributions for sums of independent random variables, Addison-Wesley, Reading, Mass., 1954.

8. M. Loeve, Probability theory, Van Nostrand, Princeton, N. J., 1963.

9. J. Pickands, Maxima of stationary Gaussian processes, Z. Wahrscheinlichkeitstheorie und Verw. Gebiete 7 (1967), 190-223.

10. D. Slepian, The one sided barrier problem for Gaussian noise, Bell System Tech. J. 41 (1962), 463-501.

11. V. A. Volkonski and Yu. A. Rozanov, Some limit theorems for random functions. II, Teor. Verojatnost. i Primenen. 6 (1961), 202-215= Theor. Probability Appl. 6 (1961), 186-198.

Virginia Polytechnic Institute, BLACKSBURG, VIRGINIA 